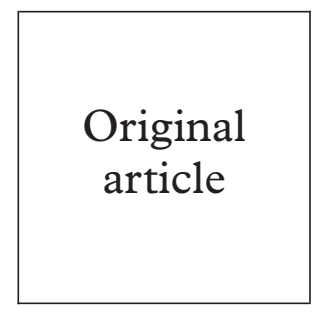

\section{HIV associated culture proved tuberculosis has increased in north central London from 1990 to 1996}

Duncan Churchill, Margaret Hannan, Robert Miller, Ian Williams, Mark Nelson, Emil Kupek, Richard Coker

Objectives: To examine rates of culture proved tuberculosis in HIV infected patients in three specialist centres in north central London.

Methods: Cases of tuberculosis in patients with previously documented HIV infection from 1990 to 1996 were identified retrospectively from microbiological/clinical records at Chelsea and Westminster, St Mary's, and University College London Hospitals.

Results: Between 1990 and 1996202 cases of culture proved tuberculosis were identified at the three centres. Of these, 132/202 (65.3\%) occurred in homosexual/bisexual men, 41/202 (20.3\%) were in patients with heterosexual contact in sub-Saharan Africa, and 29/202 (14.4\%) were in "others." Overall 148/202 (73.3\%) had pulmonary tuberculosis. The total number of HIV infected individuals seen at the three centres increased from 4298 in 1990 to 5048 in 1996. Rates of tuberculosis in the three centres increased from $0.46 \%$ in 1990 to $0.83 \%$ in 1996 . Part of this increase was due to an increase in tuberculosis among Africans from 1993 to 1996.

Conclusions: Rates of HIV associated tuberculosis increased in these three centres in north central London between 1990 and 1996. In part this was due to an increase in the number of African patients with HIV infection attending the three centres. In addition, there was circumstantial evidence of recent transmission among homosexual men with HIV infection. Prospective "real time" surveillance of tuberculosis in HIV infected patients is needed in order to detect case clustering and to improve tuberculosis control.

(Sex Transm Inf 2000;76:43-45)

Keywords: tuberculosis; HIV; nosocomial transmission; multidrug resistant tuberculosis
Department of

Medicine/Communicable Diseases and Public

Health, Imperial

College School of

Medicine at St Mary's

Hospital, London

W2 1PG

D Churchill

E Kupek

R Coker

Departments of Microbiology and HIV Medicine, Chelsea and

Westminster Hospital, London SW10 9NH

M Hannan

M Nelson

Department of Sexually Transmitted Diseases, Windeyer Institute of Medical Sciences, Royal Free and University College

Medical School,

Mortimer Market

Centre, London

WC1E 6AU

R Miller

I Williams

Correspondence to: Dr R F Miller

Accepted for publication 1 November 1999

\section{Introduction}

Before 1988 there was a pattern of declining incidence of pulmonary tuberculosis in England and Wales. Since 1988 this decline has reversed such that between 1988 and 1993 approximately 8000 more cases of tuberculosis were notified than would have been anticipated had the previous secular trend continued. ${ }^{1}$ Increasing socioeconomic deprivation, changes in patterns of immigration, and improved notification of tuberculosis have all been suggested as causes of this increase in incidence of tuberculosis. HIV infection is also a possible factor. The rise in tuberculosis in the United States in the mid 1980s was due in part to the high prevalence of HIV infection. ${ }^{2}$ Previous studies in the United Kingdom have shown little evidence of a major overlap between HIV infection and tuberculosis. ${ }^{3}$ In this study we examined rates of culture proved tuberculosis in HIV infected patients attending three specialist centres in north central London.

\section{Methods}

We retrospectively studied rates of tuberculosis in adults with HIV infection between 1990 and 1996 at three specialist centres in north central London (Chelsea and Westminster, St Mary's, and University College London Hospitals). In order to eliminate bias due to changes in notification practices, microbiological records at each hospital were used to identify cases of Mycobacterium tuberculosis from whom the organism was cultured from any site, rather than records of notified cases. These records were then cross referenced with HIV clinic records to identify patients known to be HIV infected at the time of diagnosis of tuberculosis. Risk factors for HIV infection in each patient were recorded.

Records from each centre were cross checked to eliminate duplication: patients seen at more than one centre during a course of treatment for tuberculosis were counted only once. Patients re-presenting with culture proved tuberculosis after completion of a course of chemotherapy lasting $\geqslant 6$ months were regarded as having two separate episodes of infection.

The total number of HIV infected patients attending the three centres in each year of the study was ascertained. This figure may overestimate the number of patients seen as some patients are known to attend more than one centre in any given year. We assumed that transfers between centres remained stable over the study period. Data on the total reported cases of AIDS from all three centres were obtained from the Public Health Laboratory Service Communicable Disease Surveillance Centre.

\section{Results}

During the study period 202 cases of culture proved tuberculosis were diagnosed in HIV infected patients at the three centres, of whom 
Table 1 Annual rates of tuberculosis in HIV infected patients attending the three centres from 1990 to 1996

\begin{tabular}{llll}
\hline Year & $\begin{array}{l}\text { No with culture proved } \\
\text { tuberculosis }\end{array}$ & $\begin{array}{l}\text { No with HIV attending } \\
\text { the three centres }\end{array}$ & $\begin{array}{l}\text { TB rate in those with HIV } \\
\text { infection (\%) }\end{array}$ \\
\hline 1990 & 20 & 4298 & 0.46 \\
1991 & 30 & 4649 & 0.64 \\
1992 & 31 & 4854 & 0.64 \\
1993 & 19 & 4776 & 0.40 \\
1994 & 25 & 4777 & 0.52 \\
1995 & 35 & 4821 & 0.72 \\
1996 & 42 & 5042 & 0.83 \\
\hline
\end{tabular}

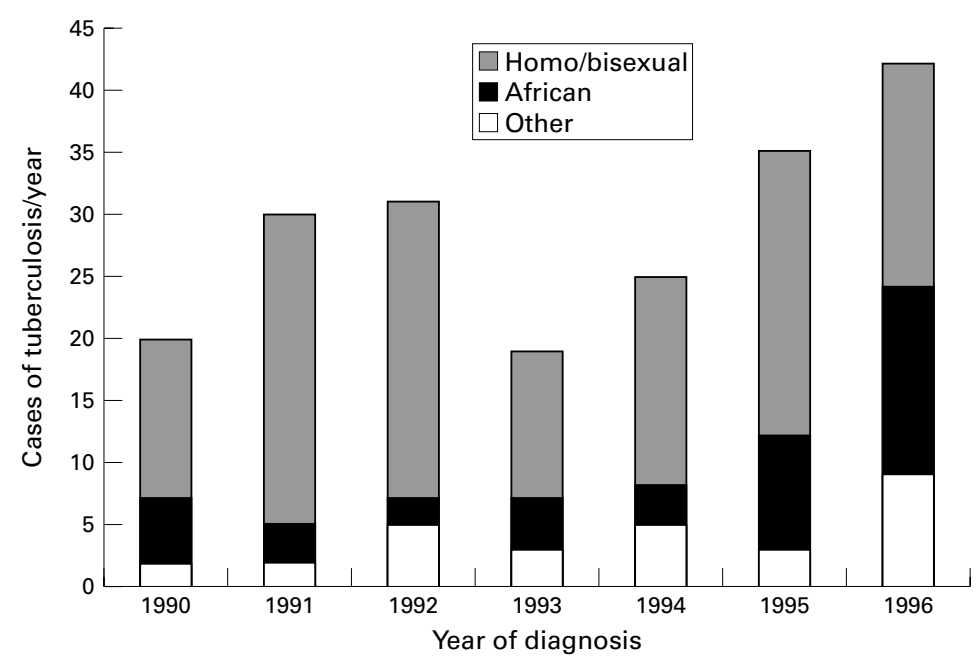

Figure 1 Number of cases of culture positive tuberculosis each year, by risk factor for HIV infection.

\section{Discussion}

We found a significant increase in rates of tuberculosis over the period of this study. We believe that this represents a true rise in rates of tuberculosis in patients with HIV infection attending these three centres, but considered several other explanations for the increase in cases. Notification of cases of tuberculosis from HIV centres has previously been poor. ${ }^{5}$ Improved notification may cause an apparent increase in tuberculosis in patients with HIV infection, and may also have led to more widespread HIV testing of patients with tuberculosis. ${ }^{5}$ Indeed, notifications of tuberculosis in North Thames rose from 1310 cases in 1990 to 1795 cases in 1995 (J Watson, personal communication). In order to minimise bias due to changes in notification practice we included in our analysis only cases of tuberculosis proved by culture. Another explanation for our data is that an increased number of patients were seen each year in the three centres. Since the total number of HIV infected patients at these centres increased by $<18 \%$ during the entire study period, this accounts for only a small part of the increase in cases.

Others have shown that, in London, Africans with HIV infection have a high incidence of tuberculosis. ${ }^{6}$ We found an increase in the number of Africans with HIV and tuberculosis from 1993 and 1996. This explains part of the overall increase of tuberculosis; nevertheless, only $20 \%$ of the cases of tuberculosis in our study occurred in Africans. Some of the observed increase in tuberculosis in HIV infected Africans may be due to more frequent HIV testing in patients presenting with tuberculosis. In contrast, almost two thirds of cases of tuberculosis in our study occurred in homosexual men. Studies from the United States which used restriction fragment length polymorphism (RFLP) analysis and epidemiological case finding show that over half the cases of tuberculosis in patients with HIV infection were clustered and, thus, were due to recently acquired infection. ${ }^{78}$ Although we did not perform routine RFLP typing of isolates of $M$ tuberculosis we are aware of several outbreaks of tuberculosis that occurred in our units during the study period..$^{9-11}$ The increase in tuberculosis in 1995 was in part due to an outbreak of nosocomial MDR tuberculosis. The peak of tuberculosis in homosexual men in 1991 and 1992 may also have been due to undetected recent transmission of tuberculosis. This peak was seen in all three units, so nosocomial transmission in a single centre would not account for the higher rate of tuberculosis observed in these years. Of note, the increased rate of tuberculosis in HIV infected patients was not recognised in any of the three centres until this study was performed. This highlights the need for better systems of surveillance of HIV associated tuberculosis.

Rates of tuberculosis in patients with HIV infection may reflect the effectiveness of general tuberculosis control programmes, providing an "early warning" system of poor tuberculosis control. ${ }^{12}$ There is a need for regional databases of patients with tuberculosis, including data on notified by the three centres, representing $31 \%$ of all cases notified in England and Wales over this period. 
HIV status and drug resistance patterns linked to prospective molecular typing of isolates to enable rapid detection of case clustering/ nosocomial transmission and to contain the threat of MDR tuberculosis.

We thank Frederick Marais, Ginny Gleissberg, Dr Alex Pym, and Dr Ian Taylor for help with the data collection; and Dr Barry Evans, Dr John Watson, Amanda Wright, and Jackie nicable Disease Surveillance Centre for providing data on AIDS and TB notifications.

Contributors: $\mathrm{RC}$ initiated this project; $\mathrm{DC}, \mathrm{MH}$, and $\mathrm{RM}$ were

Contributors: $\mathrm{RC}$ initiated this project; DC, $\mathrm{MH}$, and RM were
responsible for data collection; DC wrote the first draft; and RM responsible for data collection; DC wrote the first draft; and RM wrote the final draft of the paper. All authors were involved in
design of the study and commented on interim drafts: all reviewed the final manuscript.

Conflicts of interest: none

Funding: none.

1 Hayward AC, Watson JM. Tuberculosis in England and Wales 1982-1993: notifications exceeded predictions. Commun Dis Rep 1995;5:R29-33.

2 Centers for Disease Control and Prevention. Tuberculosis morbidity-United States 1992. MMWR 1993;42:696-7.

3 Watson JM, Meredith SK, Whitmore-Overton E, et al. Tuberculosis and HIV: estimates of the overlap in England and Wales. Thorax 1993;48:199-203.
4 Kumar D, Watson JM, Charlett A, et al on behalf of a Public Health Laboratory Service/British Thoracic Society/ Department of Health Collaborative Group. Tuberculosis in England and Wales in 1993: results of a national survey. Thorax 1997;52:1060-7.

5 Pym AS, Churchill DR, Gleissberg V, et al. Reasons for increased incidence of tuberculosis. Audit suggests that undernotification is common. BM7 1995;311:570.

6 Del Amo J, Petruckevitch A, Phillips AN, et al. Spectrum of disease in Africans with AIDS in London. AIDS 1996;10: 1563-69.

7 Alland D, Kalkut GE, Moss AR, et al. Transmission of tuberculosis in New York City-an analysis by DNA fingerprinting and conventional epidemiological methods. N Engl f Med 1994;330:1710-6.

8 Small PM, Hopewell PC, Singh SP, et al. The epidemiology of tuberculosis in San Francisco-a population-cased study using conventional molecular methods. $N$ Engl f Med 1994;330:1703-9.

9 Outbreak of hospital acquired multidrug resistant tuberculosis. Commun Dis Rep 1995;5:161.

10 Kent PJ, Uttley AHC, Stoker NG, et al. Transmission of tuberculosis in British centre for patients infected with HIV BMF 1994;309:639-40.

11 De Cock KM, Miller R, Zumla A, et al. Nosocomial transmission of tuberculosis in HIV/AIDS units in London. Genitourin Med 1997;73:322.

12 Coker R, Miller R. HIV-associated tuberculosis. BMF 1997; 314:1847. 